\title{
Results of Gamma Ray and Neutrino Search by Yakutsk Array Complex Data
}

\author{
Stanislav Knurenko*i \\ $Y$ u. G. Shafer Institute of Cosmophysical Research and Aeronomy \\ E-mail: knurenkodikfia.ysn.ru

\section{Igor Petrov} \\ Yu. G. Shafer Institute of Cosmophysical Research and Aeronomy \\ E-mail: igor.petrov@ikfia.ysn.ru
}

\begin{abstract}
To search for photon produced air showers complex analysis of the Yakutsk array data of showers with energy more than $5 \cdot 10^{18} \mathrm{eV}$ was carried out. Selection criteria were obtained based on simulations and experimental data. A number of air shower events with parameters close to those produced by primary photon were selected. These data allowed us to obtain an upper limit of integral photon flux in ultra-high energy cosmic radiation.

Multiparametric analysis of air showers with energy more than $5 \cdot 10^{18} \mathrm{eV}$ and zenith angles more than 70 degrees did not find neutrino induced air showers.
\end{abstract}

36th International Cosmic Ray Conference -ICRC2019-

July 24th - August 1st, 2019

Madison, WI, U.S.A.

* Speaker.

${ }^{\dagger}$ A footnote may follow. 


\section{Introduction}

The nature of generation of cosmic rays of ultrahigh energies (above $10^{18} \mathrm{eV}$ ) in the Universe is still unknown [1, 2]. According to models $[3,4,5]$, primary photons of high and ultrahigh energies and astrophysical neutrinos are expected to exist in cosmological space, along with other particles (nuclei of various chemical elements). The expected flux of cosmic rays of ultrahigh energies, near the Earth, will depend on nature, the degree of propagation and spatial distribution of astrophysical sources $[6,7]$.

Photons do not deflect from their original direction because it charge is neutral and for this reason are good for searching point sources generating ultra-high energy particles. For this reason, the photon component can be used to determine the physical characteristics of cosmic ray sources and study the interaction of primary photons of ultrahigh energy with the photon field of the Universe and, as a consequence, to explain the shape of the cosmic-ray spectrum in the energy range $10^{19}-10^{20} \mathrm{eV}[8,9,11,12]$.

Neutral particle produced air showers due to their physical properties can go a long way in the matter and their depth of the maximum development near sea level, i.e. "young showers". The base of such air shower is electron-photon component, which scatters to big angles and has big delay relative to particles in the shower core. Hence, in such shower events one needs to expect a large number of peaks from electrons, positrons and photons on the signal scan from scintillation detector $[13,14,15]$.

\section{Experimental Data}

In order to study characteristics of EAS development from primary gamma ray we modelled longitudinal development of air showers to the sea level using CORSIKA program [5]. Air shower parameters were calculated by QGSjetII-04 [6, 7, 8] with thinning, in the case of energy higher than $10^{18} \mathrm{eV}$. In the model we took into account registration condition of each component including response of each detectors and experimental data analysis.

Experimental data of electron-photon component of air shower with $\mathrm{E} \geq 1 \mathrm{EeV}$ obtained by continuous and long-term observation at the Yakutsk array [9]. Radial development of charged particles on the plane of the array registered by 150 ground scintillation detectors with area of $2 \mathrm{~m}^{2}$ each and 125 underground scintillation detectors with the same size. Longitudinal development was reconstructed by the 50 Cherenkov light detectors $[10,11,12,13]$.

In Fig. 1 calculations shown against EAS fraction of muon using density of muon flux at the distance $600 \mathrm{~m}$ from the axis. From Fig. 1 it's seen that average distribution of each component of cosmic radiation allocates in certain regions and can be distinguished in the experiment with high enough accuracy of the muon measurements [13]. As can be seen from the model calculations gamma ray produced air shower has significantly less muons than in proton or iron induced air showers. With accuracy of measurement of $\rho_{\mu}(600)$ at 5-8\% level it is possible to distinguish gamma ray induced air shower.

Longitudinal development of the shower at the Yakutsk array is reconstructed by the data of registration of the Cherenkov light $[14,15]$, using the mathematical apparatus used in solving inverse problems $[12,16]$. 


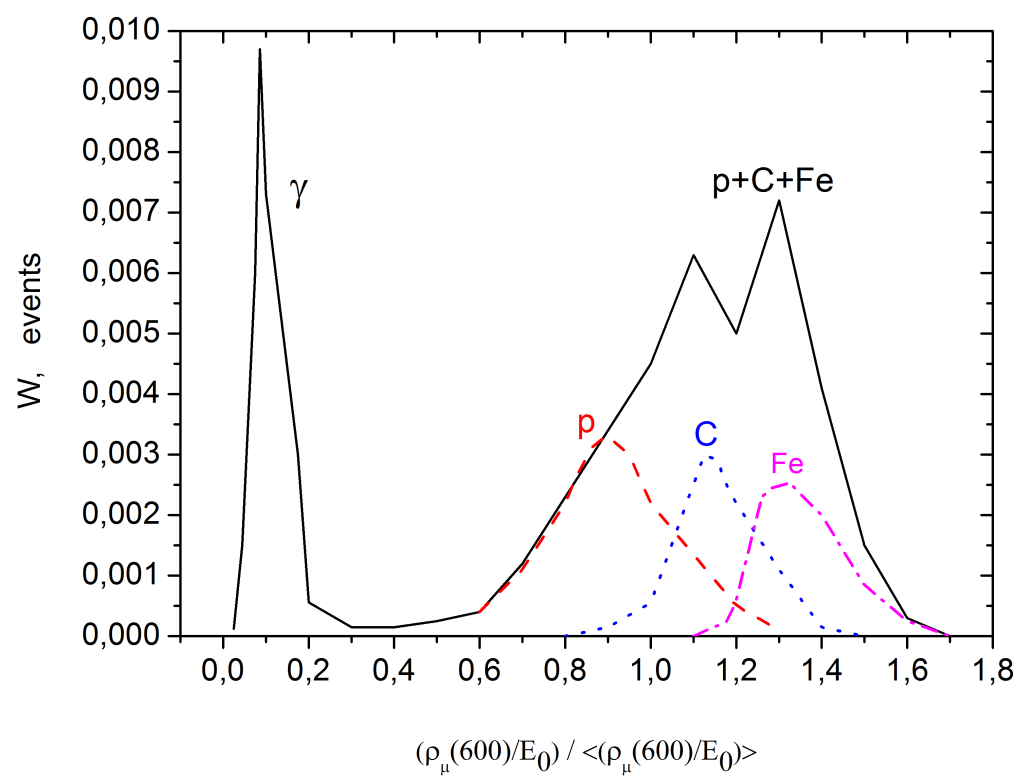

Figure 1: Distribution of showers with energies above $10^{18} \mathrm{eV}$ and zenith angle $\theta \leq 70^{\circ}$. The curves are gamma ray $\gamma$ (solid), proton $\mathrm{p}$ (dash), carbon $\mathrm{C}$ (dot) and iron nucleus Fe (dash-dot) as simulated with QGSjetII-04 [6, 8]

Using the database of Cherenkov data, the distribution of $\mathrm{X}_{\max }$ from the primary energy of the shower was obtained (see Fig. 2). It can be seen from Fig. 2 that in the distribution there are cases of EAS with $\mathrm{X}_{\max } \geq 800 \mathrm{~g} / \mathrm{cm}^{2}$, i.e. have a low maximum development of the shower in relation to the events formed by the iron nucleus and even the proton. These showers formed the basis for this analysis.

Gamma ray produced shower consist small number of muons. If the array detects muons then on number of muons in the shower one can judge the nature of the primary particle i.e. its atomic weight. We can assume this from calculations of muon components by hadron interaction models shown in Fig. 1 [14, 17].

At the Yakutsk array the fraction of muons in the shower determined by relation of muon flux density at distances of $600 \mathrm{~m}$ and $1000 \mathrm{~m}$ to the total charged component $\rho_{\mu} / \rho_{\mu+e}$, since these parameters are measured with better accuracy than the total number of muons $\mathrm{N}_{\mu}$ and charged particles $\mathrm{N}_{\mu+e}$ in showers with total energy $\mathrm{E}_{0} \geq 10^{18} \mathrm{eV}$.

It is well known that muon number depends on the height of maximum development of air showers in the atmosphere. That is why a fraction of muons very sensitive to $\mathrm{X}_{\max }$ of cascade curve. And the fraction of muons from the proton and iron nucleus, as it follows from Fig. 3, is localized in different places, which makes it possible to divide showers into protons and showers from the iron nucleus in this parameter. And that feature was used in the present paper to search for neutral particles in cosmic rays.

For this, we used the experimental dependence of $X_{\max }$ on $\rho_{\mu}(600) / \rho_{s}(600)$, shown in Fig. 3. The fits are calculations made using QGSjetII-04 for different zenith angles.

Air showers produced by different primary particles have a maximum development at different 


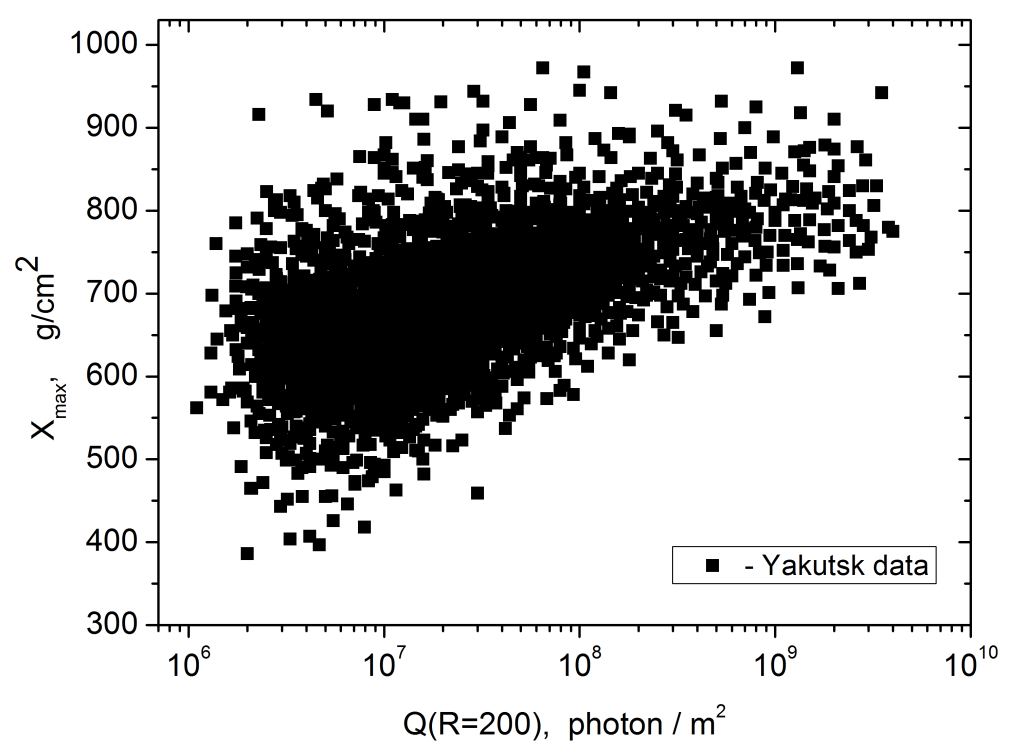

Figure 2: Dependence of $X_{\max }$ from classification parameter Q (200) - EAS Cherenkov light flux density at a distance of $200 \mathrm{~m}$ from the shower axis. Data obtained in 1973-2014

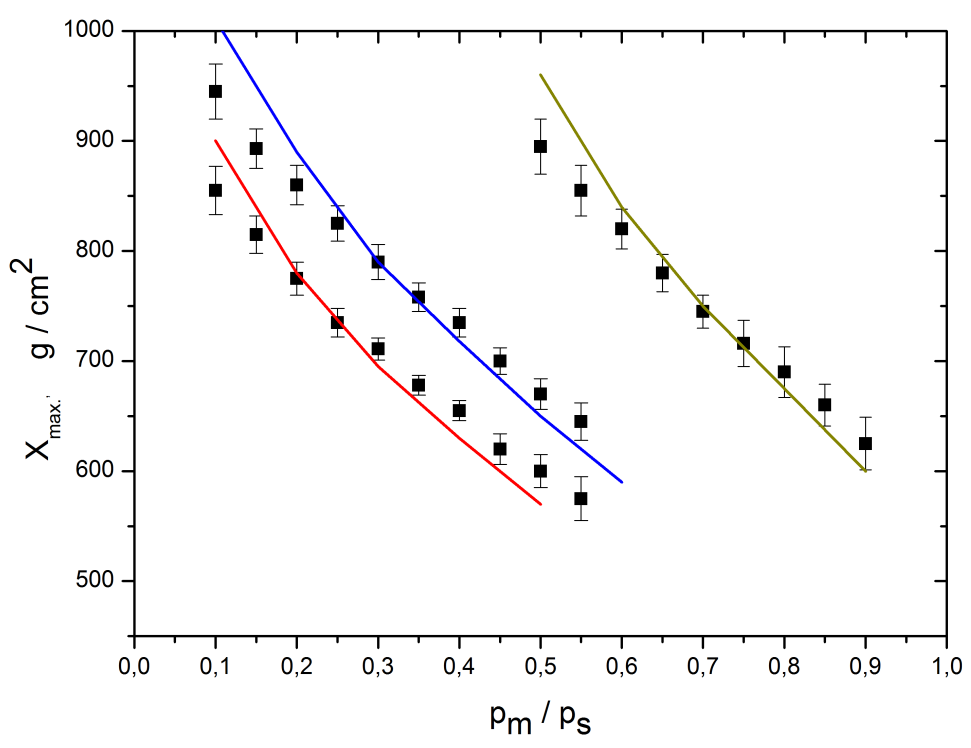

Figure 3: Dependence of muon fraction on depth of maximum development of electron-photon cascade of air showers at zenith angles $\theta_{1}=18^{\circ}, \theta_{2}=38^{\circ}$ and $\theta_{3}=58^{\circ}$. The fits are made for QGSJetII-04 model 
Table 1: Effective exposure of the Yakutsk array

\begin{tabular}{|c|c|c|c|}
\hline $\mathrm{E}_{0}, \mathrm{eV}$ & $\mathrm{K}_{1}, \%$ & $\mathrm{~K}_{2}, \%$ & $\mathrm{~S}_{\text {eff. } \gamma} \cdot T \cdot \Omega$ \\
\hline $1 \cdot 10^{18}$ & 6.6 & 6.6 & 153.9 \\
\hline $3 \cdot 10^{18}$ & 7.5 & 7.5 & 210.6 \\
\hline $6 \cdot 10^{18}$ & 12.0 & 12.0 & 555.1 \\
\hline $9 \cdot 10^{18}$ & 15.2 & 15.2 & 853.5 \\
\hline
\end{tabular}

depth in the atmosphere. Because of this, part of secondary particles (mostly electrons) loses energy to ionization of the air and eliminated from the cascade process. Then on the sea level will arrive a certain type of particles: electrons, photons, muons in the case of inclined shower and only muons in the case of strongly inclined showers, which can be seen in the signal time sweep of scintillation detectors. For the primary gamma ray and neutrino, depth of maximum is going to be near the level of observation and we can expect scintillation detector response inherent in the electron-photon component of the shower.

\section{Estimation of gamma-rays flux upper limit}

To search for EAS produced by ultra-high energy photons we concluded following criteria, which the shower should satisfy to be considered as a shower produced by a neutral particle:

- The depth of the maximum $\mathrm{X}_{\max }$ of the air shower must be within $850-950 \mathrm{~g} / \mathrm{cm}^{2}$ range;

- The fraction of muons must be $\leq 0.1$ (Fig. 1) [17, 18, 19, 20, 21];

- There must be multi-peak structure on the scan of the signal of scintillation detector, specific for "young" showers [4];

The aperture of the Yakutsk array for ultra-high energy showers for the selected time period was equal to:

$$
S_{\text {geom }} \cdot T \cdot \Omega=949.54 \mathrm{~km}^{2} \cdot \mathrm{sr} \cdot y
$$

Effective exposure after implementation of coefficients shown in table 1.

The implementation of the coefficients made possible to take into account the dependence of $\mathrm{S}_{e f f . \gamma}$ on the zenith angle and the EAS trigger with the shower selection threshold equal to 2 particles / $\mathrm{m}$. In this case, the probability of detecting showers over charged particles was equal to $\mathrm{W}_{p} \geq 0.95$, and the accuracy of the EAS parameters being determined was not worse than $10 \%$.

An upper limit on a mathematical expectation of number of photons is determined following [22] for 95\% CL and number of candidates, assuming to be produced by primary photon of ultrahigh energies table 2.

The integral flux upper limit for primary gamma-ray follows from the formula:

$$
F_{\gamma}^{95 C L}=\left(n_{\gamma}\left(E_{\gamma}>E_{0}\right)\right) / S_{e f f \cdot \gamma} \cdot T \cdot \Omega
$$


Table 2: Upper limit of the photon flux

\begin{tabular}{|c|c|c|c|c|}
\hline Energy, eV & $1 \cdot 10^{18}$ & $3 \cdot 10^{18}$ & $6 \cdot 10^{18}$ & $9 \cdot 10^{18}$ \\
\hline $\mathrm{N}_{\gamma}$ & 4 & 2 & 3 & 2 \\
\hline $\mathrm{S}_{\text {eff. } \gamma}$ & 153.9 & 210.6 & 555.1 & 853.5 \\
\hline $\mathrm{n}_{\gamma}$ & 9.76 & 6.72 & 8.25 & 6.72 \\
\hline $\mathrm{F}^{95 C L}<$ & 0.063 & 0.032 & 0.015 & 0.08 \\
\hline
\end{tabular}

Thus we obtained integral flux upper limit for photons within 1-10 EeV (Fig. 4)

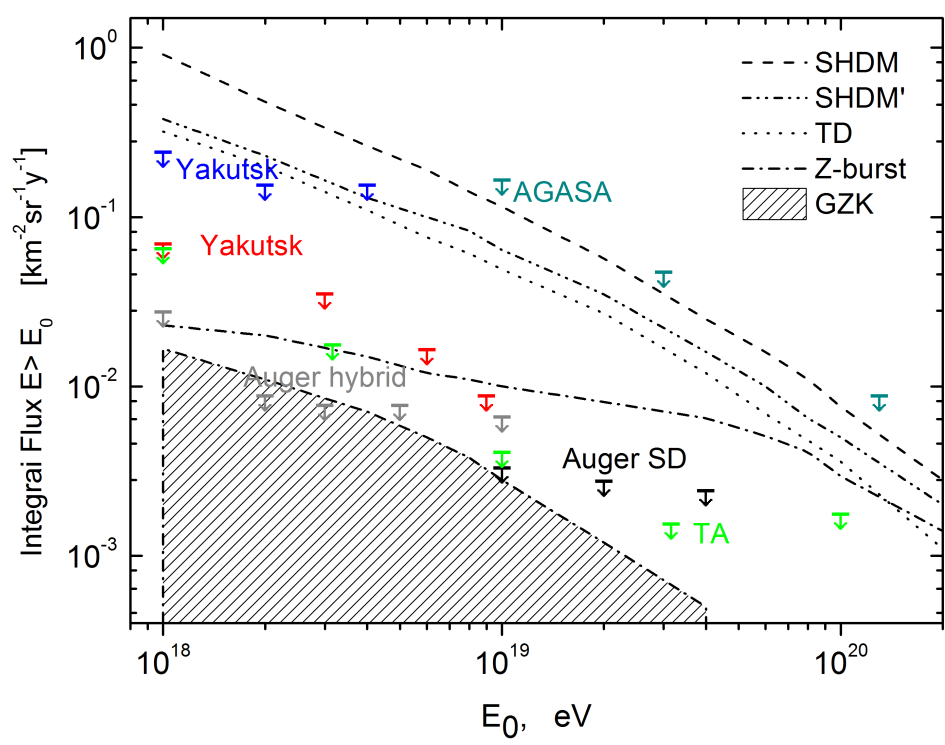

Figure 4: Photon flux limit by the Yakutsk Array data: new [26] and old [27]. Comparison with the Auger [28], AGASA [29], TA [30] and the predictions of certain models [23, 24, 25, 31]

Fig. 4 shows predictions of certain models for integral flux of primary gamma-rays, which indicates possible sources [23, 24, 25]. Those are super heavy dark matter (SHDM) [23] and SHDM' [25], topological defects (TD) and Z-bursts [23]. The first experimental results obtained at AGASA, Yakutsk (old) and Auger rather indicated on SHDM and SHDM' while newer results obtained lately including new Yakutsk array results on gamma-ray sources closer to GZK mechanism $[23,31]$.

The difference between early results and new ones is associated with an increase in exposure and models that were used to estimate the flux of photons in cosmic rays from the experimental data of EAS. The refinement of the number of muons in the new models in the direction of their increase caused a change in the selection criteria for showers and, consequently, a decrease in the integral flux of the primary gamma ray.

Hence, based on the results of the work, we obtained empirical estimations of integral flux 
upper limit of photons in cosmic rays with energies $\mathrm{E} \geq 1 \mathrm{EeV}$.

\section{Conclusion}

Multicomponent analysis of EAS using the above criteria did not reveal explicit showers produced by a gamma-ray or neutrino [32]. At the same time, calculations based on the QGSJETII model [33] for the primary proton, iron nucleus, and gamma-ray lead to the conclusion that if we take into account the fluctuations in the muon measurements within the $1 \sigma$ range, the probability of recording the EAS from there are neutral particles. Candidates for such showers can be considered as the "muonless" showers found in the Yakutsk EAS array [34]. The presented results (Fig. 4) on the upper limit of the gamma-ray flux can be used to test various astrophysical models (including the search for dark matter) and to establish the nature of the formation of particles such as astro-neutrinos in cosmological space.

In order to study the nature of neutral particles in the region of ultrahigh energies, it is required to improve the method of measurements, primarily, strongly inclined air showers. it is even possible to create specialized arrays, for example, muon telescopes with a large receiving area for registering the muon fraction with an accuracy of 3-5\% in individual EAS events.

\section{Acknowledgments}

The reported study was funded by RFBR according to the research project 16-29-13019.

\section{References}

[1] S. Knurenko, Z. Petrov and Yu. Yegorov, J. Phys. Conf. Ser. 409012090 (2013)

[2] S.P. Knurenko and A. Sabourov, in Proc. of the 33rd ICRC2013, Rio de Janeiro, 2013 [0055]

[3] G.I. Rubtsov, M. Fukushima, D. Ivanov, B.T. Stokes, G.B. Thomson and S.V. TroitskyEPJ WEb Conf. 53, 05001 (2013)

[4] S. Knurenko, Z. Petrov, Yu. Yegorov and N. Dyachkovsky, Proc 21st ECRS, Kosice (Slovakia), 465 (2008) (2003)

[5] D. Heck, J. Knapp, J.N. Capdevielle, G. Schatz and T. Thouw, Forschungszentrum Karlstruhe Thechnical Report 6019, 90 (1998)

[6] S.P. Knurenko and A.V. Sabourov, Nucl Phys. B, 196, 319 (2009)

[7] T. Pierog and K. Werner, Phys. Rev. Lett. 101, 171101 (2008)

[8] S.S. Ostapchenko, Nucl. Phys. B, 151, 143 (2006)

[9] V.P. Artamonov, B.N. Afanasyev, A.V. Glushkov, A.A. Mikhailov, S.P. Knurenko Bull. of RAoS. Physics 58, 92 (1994)

[10] M.N. Dyakonov, S.P. Knurenko, V.A. Kolosov et al., Moscow: Science, 339 (1990)

[11] M.N. Dyakonov, S.P. Knurenko, V.A. Kolosov et al., NIM A 248, 244 (1986)

[12] S. Knurenko, V. Kolosov, Z. Petrov et al., Proc. 27th ICRC, Hamburg, 1, 177 (2001) 
[13] V.B. Atraskevich, N.N. Kalmykov, G.B. Christiansen, JETP Lett. 33, 236 (1981)

[14] G.K. Garipov, V.M. Grigoryev, N.N. Efremov et al. Proc. 27th ICRC, Hamburg 1, 885-887 (2001)

[15] S.P. Knurenko, V.A. Kolosov, Z.E. Petrov et al., Proc. 27th ICRC, Hamburg 1, 559-561 (2017)

[16] A. Tikhonov, V. Arsenin, Solution of Ill-posed Problems (Winston, NY, 1977) 258

[17] S.P. Knurenko, A.A. Ivanov, M.I. Pravdin, A.V. Sabourov, and I.Ye. Sleptsov, Nucl. Phys. B. (Pros. Suppl.)175-176, 201 (2008)

[18] S.P. Knurenko, V.P. Egorova, A.A. Ivanov, V.A. Kolosov, I.T. Makarov, Z.E. Petrov, I.Ye. Sleptsov, and G.G. Struchkov, Nucl. Phys. B. 151, 92 (2006)

[19] S.P. Knurenko and A.V. Sabourov, Nucl. Phys. B 196, 319 (2009)

[20] K. Shinozaki, M. Chikawa, M. Fukushima, N. Hayashida, K. Honda, N. Inoue, K. Kadota, F. Kakimoto, K. Kamata, S. Kawaguchi, S. Kawakami, Y. Kawasaki, N. Kawasumi, K. Mase, and S. Mizobuchi, Proc. 28th ICRC. Tsukuba (Japan) 3, 401 (2003).

[21] S.P. Knurenko, A.A. Ivanov, I.Ye. Sleptsov, Bull. of RAoS. Phys. Ser. 69, 363 (2005)

[22] G.J. Feldman and R.D. Cousins, Phys. Rev. D 57, 3873 (1998)

[23] P. Bhattacharjee and G. Sigl, Phys. Rev. 327, 109 (2000) [V. S. Berezinsky and G.T. Zatsepin, Phys. Lett. B 28(6), 423 (1969)].

[24] F. Halzen and D. Hooper, Rep. Prog. Phys. 65, 1025 (2002)

[25] J. Ellis, V.E. Mayes and D.V. Nanopoulos, Phys. Rev. D 74, 115003 (2006)

[26] S.P. Knurenko, I.S. Petrov, JETP Lett. 104, 297 (2016)

[27] A.V. Glushkov, I.T. Makarov, M. I. Pravdin, and I.E. Sleptsov, Phys. Rev. D 82, 041101 (2002).

[28] M.N. Dyakonov, A.A. Ivanov, S.P. Knurenko, V.A. Kolosov, and D.D. Krasilnikov, Proc. 17th ICRC 6, $76(1981)$

[29] K. Shinozaki, M. Chikawa, M. Fukushima, N. Hayashida, N. Inoue, K. Honda, K. Kadota, F. Kakimoto, K. Kamata, S. Kawaguchi, S. Kawakami, Y. Kawasaki, N. Kawasumi, A.M. Mahrous, and K. Mase, Astrophys. J. 571, L117 (2002)

[30] G.I. Rubtsov (for the Telescope Array Collaboration), Proceedings of Science, 551 (2017).

[31] J.K. Becker, Phys. Rep. 458, 173 (2008).

[32] S.P. Knurenko, A.K. Makarov, M.I. Pravdin, A.V. Sabourov, Bull. Russ. Acad. Sci. Phys. 75, 291 (2011)

[33] S. Ostapchenko, Phys. Lett.B 636, 40 (2006)

[34] A.V. Glushkov, I.T. Makarov, M.I. Pravdin, I.Ye. Sleptsov, D.S. Gorbunov, G.I. Rubtsov, and S.V. Troitsky, Phys. Rev. D 82, 041101(R) (2010) 